\title{
Congenital retinal macrovessel: a case report
}

\begin{abstract}
We report a rare clinical case of a 66-year-old Caucasian male with slightly decreased vision in his left eye. After comprehensive ophthalmic examination and multimodal imaging with fundus photography, spectral domain optical coherence tomography (OCT), fluorescein angiography (FA), and a 30-2 visual field tests, he was diagnosed with congenital retinal macrovessel crossing the fovea avascular zone in his left eye. His right eye is unremarkable on ophthalmic exams. Congenital retinal macrovessel refers to an aberrant vessel, usually a vein, which transverse the macula and supplies both sides of the horizontal raphe. Various retinal abnormalities or clinical complications have been reported in association with congenital retinal macrovessel, such as cystoid macular edema, fovea cysts, macular exudates, hemorrhage, serous retinal detachment and branch vein occlusion. In our patient, there were not significant clinical complications. The reduction in the vision of the left eye could be explained by irregularity and slight enlargement of the foveal avascular zone (FAZ). In addition, patient's congenital macrovessel may have caused a relative angioscotoma, which could also affect the visual acuity (VA).
\end{abstract}

Volume 4 Issue 4 - 2016

\author{
Jurkute Neringa,' Cimbalas Andrius ${ }^{2}$ \\ 'Karoliniškių Clinic,Vilnius, Lithuania \\ ${ }^{2}$ Clinic of Ear, Nose, Throat, and Eye Diseases, Centre of Eye \\ Diseases, Vilnius University, Lithuania
}

Correspondence: Jurkute Neringa, Karoliniškių Clinic,Vilnius, Lithuania, Email jurkute.neringa@gmail.com

Received: May 13, 2016 | Published: July 25, 2016

Keywords: congenital retinal macrovessel, aberrant retinal vessel, fluorescein angiography

\begin{abstract}
Abbreviations: VUH SK, vilnius university hospital santariskiu klinikos; VA, visual acuity 000; IOP, intraocular pressure; BCVA, best corrected visual acuity; FA, fluorescein angiography; FAZ, foveal avascular zone; OCT, optical coherence tomography; PEX, pseudoexfoliations
\end{abstract}

\section{Introduction}

In 1869, Mauthner was first described congenital retinal macrovessel as "an aberrant retinal vessel". In 1982, Brown et al. ${ }^{1-4}$ formally termed such abnormal vessel as "congenital retinal macrovessel", which presents as unilateral, abnormally large, aberrant retinal vessel and transverses through the foveal avascular region in the posterior region. Such vessel supplies or drains the retina both inferiorly and superiorly to the horizontal raphe. Its origin often derives from the vein without a significant clinical symptom and decreased visual acuity.3,5 In rare cases, a congenital retinal macrovessel can be an artery or an artery and vein together ${ }^{1-3}$ In this article, we report a patient with a congenital retinal macrovessel crossing the foveal avascular region and touching the fovea, with a minimal visual defect and no macular thickening.

\section{Case presentation}

A 66-year-old Caucasian man was referred to the VUH SK with slightly decreased vision and suspected macular degeneration in his left eye. The patient was unaware of a vision problem until he became presbyopic with age. Three to four years ago, he noticed that his left eye vision was not as good as his right eye vision. VA was 1.0 in the right eye and his BCVA was 0.8 in the left eye. At presentation patient's IOP was $28.0 \mathrm{mmHg}$ in the right eye and $17.3 \mathrm{mmHg}$ in the left eye without taking any antihypertensive drugs. Pachymetry was $565 \mu \mathrm{m}$ in the right eye and $556 \mu \mathrm{m}$ in the left eye. Slit-lamp examinations of the anterior segments of both eyes showed PEX on the anterior surface of the lenses. Since right eye IOP was elevated and PEX on the lenses were found, patient was highly suspected for glaucoma and was followed. During the follow up period IOP fluctuated (Figure 1) in both eyes and Bimatoprost drops were prescribed for a treatment. Unfortunately, later the patient was lost to follow-up. His personal and family medical histories were unremarkable.

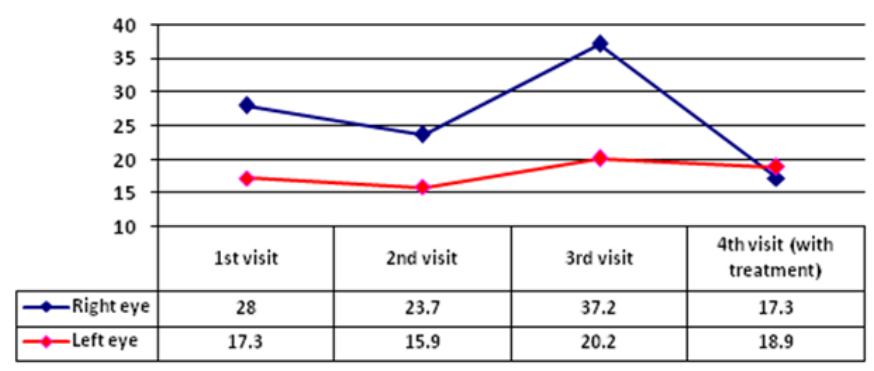

Figure I IOP fluctuation $\mathrm{mmHg}$ during the follow-up period.

The patient underwent comprehensive ophthalmic examination and multimodal imaging with fundus photography, Spectral Domain OCT (Heidelberg Engineering, GmbH, Dossenheim, Germany), FA and a 30-2 visual field test. The fundus examination of the right eye was normal (Figure 2), while the examination of the left eye revealed a retinal macrovessel crossing the macula and touching the fovea (Figure 3). FA demonstrated earlier filling of the aberrant retinal vein (Figure 4) compared with the superotemporal central retinal vein branche; many anastomoses were observed between these vessels (Figure 5). Left eyes FAZ was slightly bigger and irregular in shape compared to the right eye FAZ (Figure 6\&7). OCT examination showed subtle parafoveal thickening (average macular thickness in 5 quadrants: center circle $313 \mu \mathrm{m}$, superior $356 \mu \mathrm{m}$, temporal $360 \mu \mathrm{m}$, inferior $336 \mu \mathrm{m}$ and nasal $363 \mu \mathrm{m}$ ) without intraretinal fluid accumulation (Figures 8\&9). Right eyes 30-2 visual field test showed paracentral scotoma (Figure 10), which was associated with glaucoma, while left eyes test findings did not correlate with the fundus and OCT findings (Figure 11), however the patient's congenital macrovessel may have caused a relative angioscotoma that resulted in reduction of vision in the left eye. 


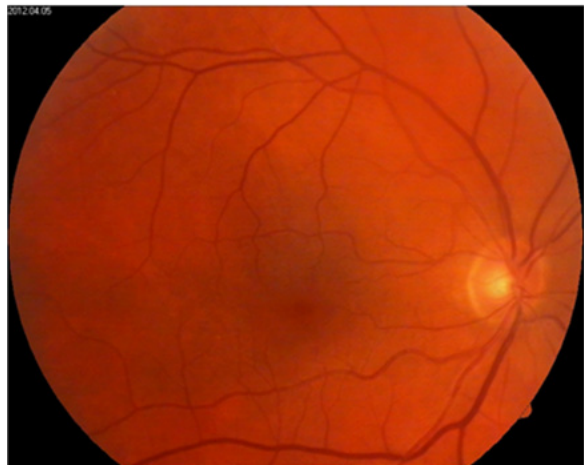

Figure 2 The fundus of the right eye was normal.

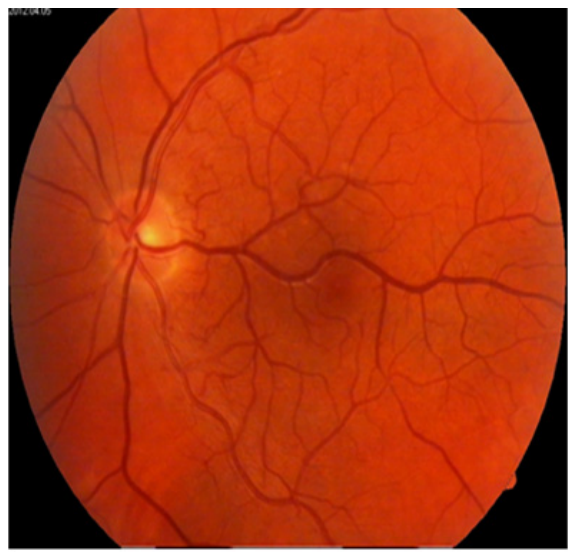

Figure 3 The fundus of the left eye revealed revealed a retinal macro vessel crossing the macula and touching the fovea.

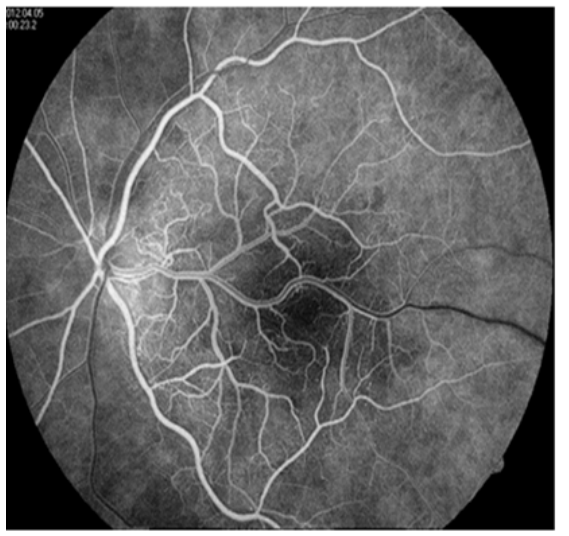

Figure 4 Early filling with Fluorescein of the aberrant retinal vein compared with the super temporal branch of the vein.

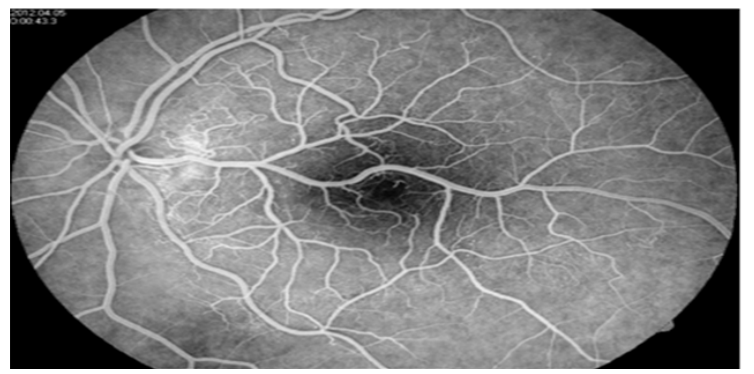

Figure 5 Anastomoses between the central retinal vein temporal branches.

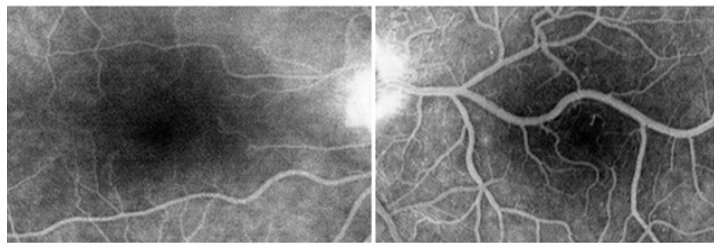

Figures 6\&7 FAZ irregularity and slight increase in size of the left eye compared to the right eye.

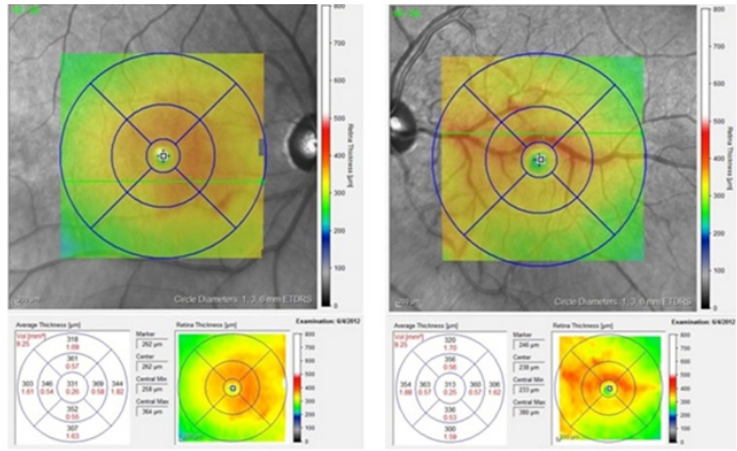

Figure 8 Subtle parafoveal thickening of the left eye compared with right eye.

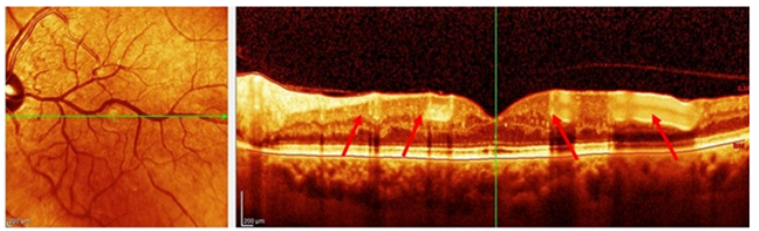

Figure 9 Normal foveal contour and the diameter of the macrovessel (arrows) in the left eye (NT section, $0^{\circ}$ ).

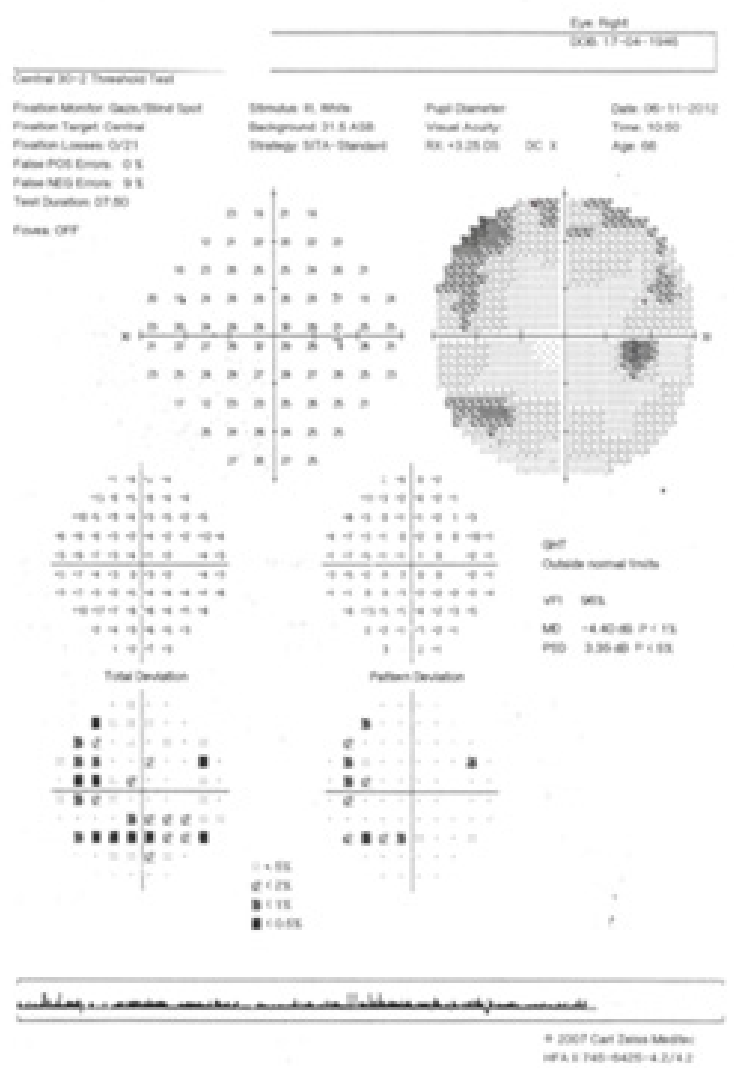




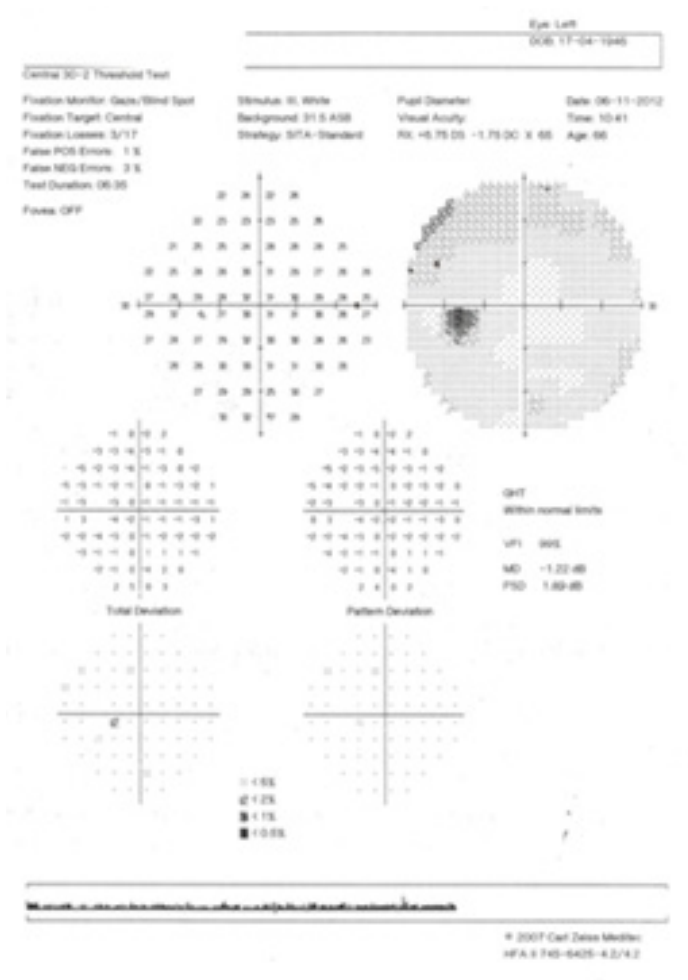

Figures I0\&II Results from a central 30-2 visual field test.

\section{Discussion}

It is believed that congenital retinal macrovessels are formed in weeks 15-16 of gestation, although the underlying cause is unknown. For some authors, the prevalence of this condition is approximately $1 / 200,000 .{ }^{3,4,6}$ Most congenital retinal macrovessel cases are unilateral and stable with excellent visual prognosis and are detected on routine examination. In rare cases, foveolar cysts, macular hemorrhages, pigmentary changes, serous macular detachment and a displaced fovea may also be seen. ${ }^{1,2,-6}$ These findings can deteriorate VA. In addition, the relative angioscotoma may cause reduction in vision. The presence of hemoglobin circulating in the vessel could explain this phenomenon. Shah et al. ${ }^{7}$ reported reduced retinal sensitivity at the macular area using liquid crystal display microperimetry in a case of congenital retinal macrovessel with no sign of macular edema on OCT. FA findings of anomalous vessels consist of early filling and delayed emptying, capillary perfusion abnormalities, focal capillary dilatations, direct arteriovenous communications and enlargement of the foveal avascular zone. ${ }^{1,3,5,8}$

In our case, the retinal macrovessel that originated in the inferotemporal central retinal vein branch crossed the macula and touched the fovea. FA showed an early filling of the aberrant retinal vein and there were many anastomoses between the inferotemporal and superotemporal central retinal vein branches without any leakage. The FAZ asymmetry was noticed between both eyes. We performed an OCT examination that showed a normal foveal contour, the diameter of the macrovessel and subtle parafoveal thickening without intraretinal fluid accumulation De Crecchio G et al. ${ }^{5}$ Reported a case of a macrovessel with decreased vision due to serous retinal detachment of the macula, macular thickening with a preserved foveal contour and distorted retinal microarchitecture on OCT. We also performed a central 30-2 visual field test to support the observed macular changes, but the findings did not correlate with the OCT findings.

\section{Conclusion}

We observed that the FA findings showed very subtle changes in the FAZ of the left eye. There were no signs of foveolar cysts, macular hemorrhages, exudates, edema, serous macular detachment or any leakage from the anastomoses. The reduction in the vision of the left eye could be explained by FAZ irregularity and slight enlargement. In addition, patient's congenital macrovessel may have caused a relative angioscotoma which could also affect the VA. In cases of congenital retinal macrovessels with reduced vision that cannot be explained by FA, OCT imaging systems are indispensable methods for clinical diagnosis and its related clinical complications in the macular region.

\section{Ethical consent}

None.

\section{Acknowledgments}

None.

\section{Conflicts of interest}

None.

\section{Funding}

None.

\section{References}

1. Brown GC, Donoso LA, Magargal LE, et al. Congenital retinal macrovessels. Arch Ophthalmol. 1982;100(9):1430-1436.

2. Ceylan OM, Gullulu G, Akin T, et al. Congenital retinal macrovessel: atypical presentation using optical coherence tomography. Int Ophthalmol. 2011;31(1):55-58.

3. Ghasemzadeh F, Alemzadeh SH, Barkhordari HR, et al. Congenital Macular Macrovessels: A Case Report and Review of the Literature. Iranian Journal of Ophthalmology. 2010;22(3):61-5.

4. Perez-Carro G, Miranda-Rollon M, Junceda-Moreno C, et al. Congenital retinal macrovessels: a discovery by chance. Arch Soc Esp Oftalmol. 2008;83(4):273-276.

5. de Crecchio G, Alfieri MC, Cennamo G, et al. Congenital macular macrovessels. Graefes Arch Clin Exp Ophthalmol. 2006;244(9):183-187.

6. Bhatia HK, Sharma S, Laxminarayana P. Congenital Retinal Macrovessel with Normal Visual Acuity: A Case Report. Int J Ophthalmol Clin Res. 2015;2:017.

7. Shah VA, Chalam KV. Congenital retinal macrovessel causes reduced retinal sensitivity at the macula. Eur J Ophthalmol. 2004;14(4):341-344.

8. Gurwood SA, Bailey JT, Pelino JC. Congenital retinal macrovessel: A case report. Optometry. 2001;72(9):97-602. 